\title{
Toric Topographically Customized Transepithelial, Pulsed, Very High-Fluence, Higher Energy and Higher Riboflavin Concentration Collagen Cross-Linking in Keratoconus
}

\author{
Anastasios John Kanellopoulos ${ }^{a, b} \quad$ William J. Dupps, ${ }^{c, d} \quad$ Ibrahim Seven $^{\text {e, }}$ \\ George Asimellis ${ }^{a}$ \\ ${ }^{a}$ Laservision.gr Eye Institute, Athens, Greece; ${ }^{b}$ Department of Ophthalmology, NYU \\ Medical School, New York, N.Y., Departments of ${ }^{\mathrm{C}}$ Ophthalmology and ${ }^{\mathrm{d}}$ Biomedical \\ Engineering, Cleveland Clinic, ${ }^{e}$ Cole Eye Institute, Cleveland Clinic, and ${ }^{\mathrm{f} D e p a r t m e n t}$ of \\ Chemical and Biomedical Engineering, Cleveland State University, Cleveland, Ohio, USA
}

\section{Key Words}

Topography customizable cross-linking · High-fluence cross-linking ·

Transepithelial cross-linking - Toric cross-linking · Keratoconus · Photorefractive intrastromal cross-linking $\cdot \mathrm{KXL}$ II

\begin{abstract}
Purpose: To report a novel application of toric topographically customized transepithelial collagen cross-linking (CXL) aiming to achieve refractive astigmatic changes in a keratoconic cornea. Methods: Specially formulated riboflavin transepithelial administration and delivery of high-fluence UVA in a topographically customized pattern was applied in an eye with progressive keratoconus. Visual acuity, cornea clarity, keratometry, topography, and pachymetry with a multitude of modalities, as well as endothelial cell counts were evaluated for $>6$ months. Results: Uncorrected distance visual acuity changed from preoperative 20/40 to $20 / 25$ at 6 months. A mean astigmatic reduction of $0.8 D$, and significant cornea surface normalization was achieved 6 months postoperatively. There was some mild change in the epithelial distribution, with the treated area having a slight normalization in the average epithelial thickness. Conclusions: We introduce herein the novel application of a topograph-
\end{abstract}


Kanellopoulos et al.: Topographically Customizable Toric Transepithelial CXL

ically customizable transepithelial $C X L$ in progressive keratoconus in order to achieve an astigmatic refractive effect and ectasia stabilization. This novel technique offers a nonablative and nonincisional approach to treat irregular astigmatism in ectatic cornea with rapid visual rehabilitation.

(C) 2014 S. Karger AG, Basel

\section{Introduction}

Collagen cross-linking (CXL) has been employed for many years as a means of stabilizing cornea ectasia $[1,2]$. Among the multitude of treatments and technique variations applied, CXL has been well documented to almost invariably result in some central anterior corneal flattening [3-7]. This flattening has often been attributed as 'disease regression'. We present herein a custom application with a novel device, enabling the topographically customizable administration of very high-fluence CXL, applied in a specific toric pattern, in order to achieve, besides ectasia stabilization, predictable corneal astigmatic changes.

This study received approval by the ethics committee of our institution, and adhered to the tenets of the Declaration of Helsinki. Written informed consent was obtained at the time of the first study visit.

\section{Case Presentation}

We report herein a case of a 6-month postoperative evaluation of an innovative customized toric application of transepithelial cross-linking. The patient was a 37-year-old female with a preoperative clinical diagnosis of progressive moderate keratoconus of the left eye, minimum corneal pachymetry of $450 \mu \mathrm{m}$, and uncorrected distance visual acuity (UDVA) between $20 / 32$ and $20 / 25$. One day before surgery, manifest refraction was $-0.50 /-2.25 \times$ 135.

The patient was enrolled due to keratoconus progression noted over the previous year. In the past, the patient's other eye (right) had a more evident keratoconic manifestation and had been treated a few years ago with the Athens Protocol procedure [8, 9]. The left eye was not treated at that time due to the patient's age and because it was less affected; however, the progression, in combination with the patient's desire for quick visual rehabilitation played a role in the decision to treat this keratoconic eye with this novel approach.

\section{Surgical Technique}

The riboflavin penetration through the intact epithelium (transepithelial) into the stroma involved a two-step process. The first step was the application of a ParaCel solution (Avedro Inc., Waltham, Mass., USA), a slightly hypotonic specially formulated $0.25 \%$ riboflavin solution with $0.02 \%$ benzalconium chloride. Application time was 4 min with two drops approximately every minute. The aim of this mild 'abrasive' solution application was to 'open up' the epithelial cell junctions. The manufacturer cautions against administering ParaCel for over $4 \mathrm{~min}$ to minimize the risk of epithelial sloughing. The second step was the application of VibeX Xtra (Avedro Inc.), $0.25 \%$ riboflavin isotonic saline solution. Application time was 6 min, with a drop every $30 \mathrm{~s}$. With this two-step soaking process, the riboflavin passage to the anterior chamber was approximately complete in $10 \mathrm{~min}$.

The KXL II cross-linking system (Avedro Inc.) was employed for the UVA exposure. Following alignment and focusing by the surgeon, the pupillary aperture was captured and tracked by the KXL II internal tracking mechanism. UVA irradiation was administered in a 
Kanellopoulos et al.: Topographically Customizable Toric Transepithelial CXL

topographically custom designed pattern. The toric treatment pattern and orientation relative to the astigmatic axes were investigated in preclinical computational modeling studies $[10,11]$.

As shown in fig. 1, the specific toric irradiation pattern had a 'butterfly-wing' shape, extending to $5-\mathrm{mm}$, axis oriented at $129^{\circ}$ (orthogonal to the steep axis of astigmatism), with a total energy of $14.0 \mathrm{~J} / \mathrm{cm}^{2}$ applied for $10 \mathrm{~min}$ and $22 \mathrm{~s}$. Overlapped to this was a circular irradiation area extending to a 6-mm diameter, with a total energy of $4.0 \mathrm{~J} / \mathrm{cm}^{2}$ applied for 2 min and $56 \mathrm{~s}$. The UVA power was $45 \mathrm{~mW} / \mathrm{cm}^{2}$, delivered with a pulsed mode of 1 -second on and a 1-second off. During the UVA irradiation, the cornea was moistened every couple of minutes with a drop of balanced salt solution. The patient was postoperatively treated with a combination of antibiotic/corticosteroid drops for 10 days.

The patient was examined the first day, the first week, and every month for a total duration of 6 months. At each visit, the cornea was assessed with Placido topography (Topolyzer Vario; WaveLight, Erlangen, Germany) by means of curvature maps and simulated keratometry, Scheimpflug imaging (Oculyzer II; WaveLight) by means of curvature maps and simulated keratometry, anterior surface irregularity indices and keratoconus classification, autorefraction and keratometry (Nidek AR 1; Nidek Co. Ltd., Tokyo, Japan), anterior segment optical coherence tomography (OCT) [12] (RtVue-100; Optovue Inc., Fremont, Calif., USA) providing corneal pachymetry and epithelial pachymetry maps, and endothelial cell counts measurement with a confocal specular microscopy device (CellChek XL; Konan, Irvine, Calif., USA). Cornea clarity was assessed by slit-lamp examination.

\section{Results}

The entire operation was completed in $<25 \mathrm{~min}$. The patient achieved UDVA of $20 / 32$ at the first postoperative week and 20/25 at 6 months. Cornea clarity was not affected, with no postoperative haze or epithelial complications. The patient reported no pain ( 0 on a scale of $0-10$ ) and minimal discomfort during the procedure. During the immediate postoperative follow-up visits, the patient also reported no pain. At the 6-month visit, the manifest refraction was $-1.25-0.75 \times 125$, and UDVA was 20/25. Endothelial cell counts were stable, preoperatively at 2,430 cells $/ \mathrm{mm}^{2}$ and postoperatively at 2,400 cells $/ \mathrm{mm}^{2}$.

Preoperative and postoperative visual acuity, pachymetry and keratometry average data are reported in table 1. Fig. 2 and fig. 3 illustrate examples of Placido disc and Scheimpflug imaging sagittal curvature preoperative, 6-month postoperative and difference maps. A significant astigmatic reduction was noted (on average $0.8 \mathrm{D}$ ) as measured by all keratometric modalities employed in the study. Topography (fig. 2) and Scheimpflug imaging data (fig. 3) suggest that the achieved astigmatic flattening was $1.4 \mathrm{D}$ (fig. 2, fig. 3), reaching a maximum of $2.5 \mathrm{D}$ at the steepest preoperative location. Based on the Scheimpflug imaging (Amsler-Krumeich criteria) analysis [13], preoperatively, the cornea was classified for the topographic keratoconus classification of $\mathrm{KC} 1$ and the values for the anterior irregularity indices of height decentration (IHD) and surface variance (ISV) were 0.039 and 43, respectively (fig. 3). Six months postoperatively, the topographic keratoconus classification was reduced to 'poss' (possible KC), while the anterior surface irregularity indices of surface variance and height decentration were lower, with values of 32 and 0.024 , respectively, indicating a trend towards a more normalized cornea. Fig. 4 illustrates OCT-derived corneal and epithelial thickness pachymetry maps preoperatively, at 1-week and 6-month postoperatively. 
Kanellopoulos et al.: Topographically Customizable Toric Transepithelial CXL

\section{Discussion}

The significant refractive changes as a result of classic CXL [3-7, 14-16], when applying the Dresden Protocol ( $3 \mathrm{~mW} / \mathrm{cm}^{2}$ for $30 \mathrm{~min}$ ), as well as higher UVA fluence, either superficially [17], via riboflavin placed within a femtosecond laser-created pocket or ICRS channels can be established by a number of reports [18, 19]. Such flattening, which constitutes a refractive effect resulting from differential cross-linking-induced stiffening effects, has been described by a number of clinicians as 'disease regression'. We have reported on the use of higher fluence CXL as an agent for refractive stabilization in highmyopic and hyperopic LASIK [20-22], and have also recently reported significant refractive changes in astigmatic keratotomy, when 'flash' CXL was used just on the incision margins [23].

Facilitated by the development of a customizable differential UV delivery system by Avedro and the ability to perform feasibility studies with the KXL II device, the current study provides additional evidence for the potential of patterned CXL in the deliberate treatment of refractive error. We have recently reported in vivo, topographically regular and predictable central cornea flattening effects consistent with a correction of myopia of about $2.5 \mathrm{D}$ [24]. The available interim data appear promising with regard to the potential for correcting low myopic refractive errors without tissue removal in an excimer-like fashion or other previously described thermal techniques combined with CXL [25]. Myopic, hyperopic and astigmatic corrections are other novel applications that are currently under study with this technology.

The novel toric topographically customized application of cross-linking presented herein (photorefractive intrastromal cross-linking) employed preferential corneal flattening along the flat refractive axis. Six months postoperatively, a very specific reduction of astigmatism of $0.8 \mathrm{D}$ (reaching up to $2.5 \mathrm{D}$ at certain loci) was observed. The epithelial remodeling maps in the first 6 months, when compared to normal eyes [26], suggest that there was some modest change in the epithelial distribution, with the treated area having initially a slight reduction in the average epithelial thickness, and recovering at the 6-month follow-up to a more regular distribution than the preoperative distribution. The epithelial data suggest that the achieved refractive effect is not a result of epithelial remodeling, and thus may be attributed to direct stromal remodeling. As shown in the OCT pachymetry data illustrated in fig. 4, preoperatively, the difference between the minimum corneal thickness of $503 \mu \mathrm{m}$ to the pupil center corneal thickness of $535 \mu \mathrm{m}$ was $32 \mu \mathrm{m}$, while 6 months postoperatively, the minimum corneal thickness was $542 \mu \mathrm{m}$ and the center corneal thickness $557 \mu \mathrm{m}$, with a difference of $15 \mu \mathrm{m}$. Additionally, the procedure has neither an obvious corneal thinning effect nor an apparent haze following treatment.

This procedure was relatively simple for the patient and surgeon. Since it is essentially performed through the intact epithelium, the procedure required minimal postoperative adjustment of the daily activities by the patient and produced essentially no pain or discomfort even within the first few hours after the procedure. The rapid recovery and relative safety of transepithelial CXL may additionally offer the possibility to titrate the effect through two or more treatments over time as needed.

As the patient is under continuous follow-up, and as more patients are being enrolled for the procedure, we anticipate reporting on the stability of the procedure, as well as on further refinement of treatment patterns, diameter, and centration for specific topographic patterns. 
Kanellopoulos et al.: Topographically Customizable Toric Transepithelial CXL

\section{Conclusions}

We have herein introduced a novel technique based on refractive CXL application on previously untreated keratoconic cornea as a conservative management option. At 6-month follow-up, a stable reduction in astigmatism was achieved. This is a nonablative and nonincisional approach to treat irregular astigmatism in ectatic cornea with rapid visual rehabilitation.

\section{Acknowledgments}

Funded in part by NIH R01 EY023381 (W.J.D.) and an Ohio Third Frontier Innovation Platform Award (W.J.D.). Funded in part by internal Laservision.gr funds (A.J.K., G.A.)

\section{Disclosure Statement}

Alcon/WaveLight, Allegran, Avedro, i-Optics; Optovue (A.J.K.). Cleveland Clinic, Ziemer, Avedro (W.J.D.).

\section{References}

1 Dupps WJ Jr: Special section on collagen crosslinking: new hope for more advanced ectatic disease? J Cataract Refract Surg 2013;39:1131-1132.

-2 Sinha Roy A, Rocha KM, Randleman JB, Stulting RD, Dupps WJ Jr: Inverse computational analysis of in vivo corneal elastic modulus change after collagen crosslinking for keratoconus. Exp Eye Res 2013;113:92-104.

-3 Ghanem RC, Santhiago MR, Berti T, Netto MV, Ghanem VC: Topographic, corneal wavefront, and refractive outcomes 2 years after collagen crosslinking for progressive keratoconus. Cornea 2014;33:43-48.

4 Arora R, Jain P, Goyal JL, Gupta D: Comparative Analysis of Refractive and Topographic Changes in Early and Advanced Keratoconic Eyes Undergoing Corneal Collagen Crosslinking. Cornea 2013, PMID: 23974893, Epub ahead of print.

5 Hassan Z, Szalai E, Módis L Jr, Berta A, Németh G: Assessment of corneal topography indices after collagen crosslinking for keratoconus. Eur J Ophthalmol 2013;23:635-640.

-6 Touboul D, Trichet E, Binder PS, Praud D, Seguy C, Colin J: Comparison of front-surface corneal topography and Bowman membrane specular topography in keratoconus. J Cataract Refract Surg 2012;38:1043-1049.

7 Piñero DP, Alio JL, Klonowski P, Toffaha B: Vectorial astigmatic changes after corneal collagen crosslinking in keratoconic corneas previously treated with intracorneal ring segments: a preliminary study. Eur J Ophthalmol 2012;22(suppl 7):S69-S80.

8 Kanellopoulos AJ, Asimellis G: Keratoconus management: long-term stability of topography-guided normalization combined with high-fluence CXL stabilization (the Athens Protocol). J Refract Surg 2014;30:88-93.

-9 Kanellopoulos AJ, Asimellis G: Comparison of Placido disc and Scheimpflug image-derived topographyguided excimer laser surface normalization combined with higher fluence CXL: the Athens Protocol, in progressive keratoconus. Clin Ophthalmol 2013;7:1385-1396.

-10 Seven I, Dupps WJ: Patient-specific finite element simulations of standard incisional astigmatism surgery and a novel patterned collagen crosslinking approach to astigmatism treatment. J Med Dev 2013;7:040913

11 Seven I, Roy AS, Dupps WJ: Patterned corneal collagen crosslinking for astigmatism: a computational modeling study. J Cataract Refract Surg 2014, Epub ahead of print.

12 Kanellopoulos AJ, Asimellis G: Comparison of high-resolution Scheimpflug and high-frequency ultrasound biomicroscopy to anterior-segment OCT corneal thickness measurements. Clin Ophthalmol 2013;7:22392247.

-13 Kanellopoulos AJ, Asimellis G: Revisiting keratoconus diagnosis and progression classification based on evaluation of corneal asymmetry indices, derived from Scheimpflug imaging in keratoconic and suspect cases. Clin Ophthalmol 2013;7:1539-1548.

14 Greenstein SA, Fry KL, Hersh PS: Corneal topography indices after corneal collagen crosslinking for keratoconus and corneal ectasia: one-year results. J Cataract Refract Surg 2011;37:1282-1290. 


\section{Case Reports in Ophthalmology}

\begin{tabular}{l|l}
\hline Case Rep Ophthalmol 2014;5:172-180 \\
\hline DOI: $10.1159 / 000363371$ & $\begin{array}{l}\text { C 2014 S. Karger AG, Basel } \\
\text { www.karger.com/cop }\end{array}$ \\
\hline
\end{tabular}

Kanellopoulos et al.: Topographically Customizable Toric Transepithelial CXL

15 Kanellopoulos AJ: Post-LASIK ectasia. Ophthalmology 2007;114:1230.

16 Hafezi F, Kanellopoulos J, Wiltfang R, Seiler T: Corneal collagen crosslinking with riboflavin and ultraviolet A to treat induced keratectasia after laser in situ keratomileusis. J Cataract Refract Surg 2007;33:2035-2040.

17 Kanellopoulos AJ: Long term results of a prospective randomized bilateral eye comparison trial of higher fluence, shorter duration ultraviolet A radiation, and riboflavin collagen cross linking for progressive keratoconus. Clin Ophthalmol 2012;6:97-101.

-18 Kanellopoulos AJ: Collagen cross-linking in early keratoconus with riboflavin in a femtosecond laser-created pocket: initial clinical results. J Refract Surg 2009;25:1034-1037.

19 Alió JL, Toffaha BT, Piñero DP, Klonowski P, Javaloy J: Cross-linking in progressive keratoconus using an epithelial debridement or intrastromal pocket technique after previous corneal ring segment implantation. J Refract Surg 2011;27:737-743.

20 Kanellopoulos AJ, Pamel GJ: Review of current indications for combined very high fluence collagen crosslinking and laser in situ keratomileusis surgery. Indian J Ophthalmol 2013;61:430-432.

-21 Kanellopoulos AJ, Kahn J: Topography-guided hyperopic LASIK with and without high irradiance collagen cross-linking: initial comparative clinical findings in a contralateral eye study of 34 consecutive patients. J Refract Surg 2012;28(11 suppl):S837-S840.

22 Kanellopoulos AJ: Long-term safety and efficacy follow-up of prophylactic higher fluence collagen crosslinking in high myopic laser-assisted in situ keratomileusis. Clin Ophthalmol 2012;6:1125-1130.

-23 Kanellopoulos AJ: Very high fluence collagen cross-linking as a refractive enhancement of a regressed previous astigmatic keratotomy. J Refract Surg 2013;29:504-505.

24 Kanellopoulos AJ: Novel myopic refractive correction with transepithelial, very high-fluence collagen crosslinking applied in a customized pattern: early clinical results in a feasibility study. Clin Ophthalmol 2014;8:697-702.

25 Kanellopoulos AJ: Laboratory evaluation of selective in situ refractive cornea collagen shrinkage with continuous wave infrared laser combined with transepithelial collagen cross-linking: a novel refractive procedure. Clin Ophthalmol 2012;6:645-652.

26 Kanellopoulos AJ, Asimellis G: In vivo three-dimensional corneal epithelium imaging in normal eyes by anterior-segment optical coherence tomography: a clinical reference study. Cornea 2013;32:1493-1498.

Table 1. Preoperative, 1-week and 6-month postoperative visual acuity, refractive, pachymetry and keratometry data

\begin{tabular}{lllll}
\hline Vision & S (D) & C (D) & Axis $\left(^{\circ}\right)$ & UDVA \\
\hline Preoperatively & -0.50 & -2.25 & 135 & $20 / 40$ \\
At 1 week & -0.50 & -3.00 & 112 & $20 / 32$ \\
At 6 months & -1.25 & -0.75 & 125 & $20 / 25$ \\
\hline Thickness & Corneal CT $(\mu \mathrm{m})$ & Corneal TT $(\mu \mathrm{m})$ & Epithelial CT $(\mu \mathrm{m})$ & Epithelial TT $(\mu \mathrm{m})$ \\
\hline Preoperative & 535 & 503 & 50 & 43 \\
At 1 week & 570 & 541 & 45 & 42 \\
At 6 months & 557 & 528 & 51 & 46 \\
\hline Keratometry & K1 $(\mathrm{D})$ & K2 (D) & Astigmatism (D) & Axis $\left(^{\circ}\right)$ \\
\hline Preoperative & 42.1 & 44.4 & +2.3 & 130 \\
At 1 week & 42.4 & 43.9 & +1.4 & 123 \\
At 6 months & 42.2 & 43.7 & +1.4 & 120 \\
\hline
\end{tabular}

$\mathrm{S}=$ Sphere $; \mathrm{C}=$ cylinder D = diopters; $\mathrm{CT}$ = central thickness; TT = thinnest thickness; $\mathrm{K} 1$ = flat keratometry; K2 = steep keratometry. Axis corresponds to flat meridian orientation. 


\section{Case Reports in \\ Ophthalmology}

\begin{tabular}{l|l}
\hline Case Rep Ophthalmol 2014;5:172-180 \\
\hline DOI: $10.1159 / 000363371$ & $\begin{array}{l}\text { @ 2014 S. Karger AG, Basel } \\
\text { www.karger.com/cop }\end{array}$ \\
\hline
\end{tabular}

Kanellopoulos et al.: Topographically Customizable Toric Transepithelial CXL
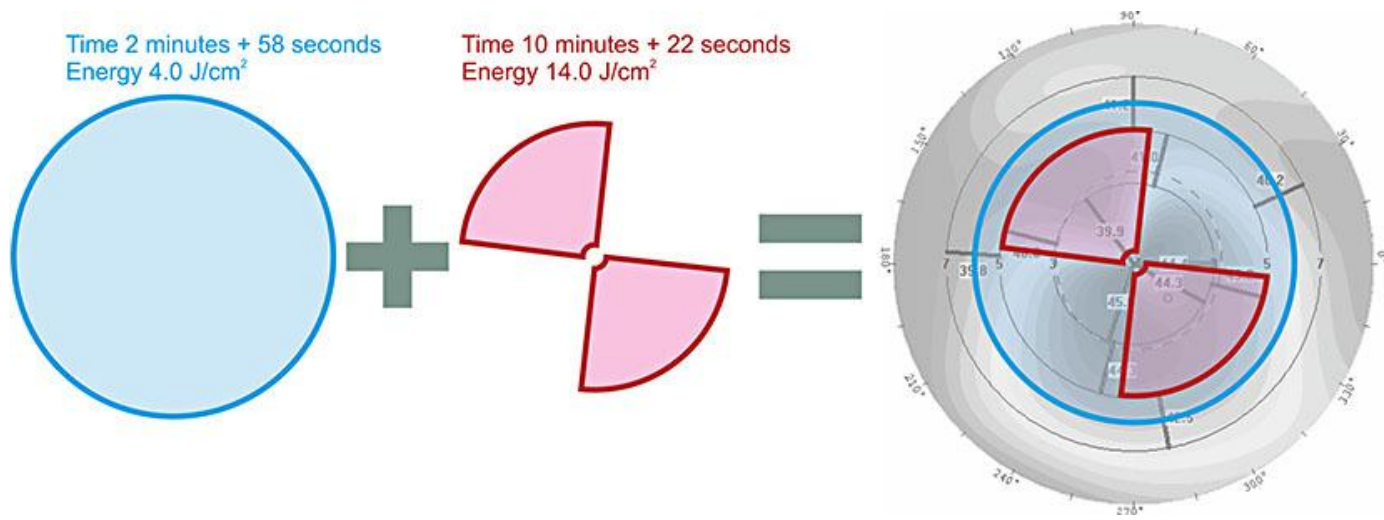

Fig. 1. Customized treatment profile employed in the treatment. Left panel: details of the applied customizable pattern and parameters for UVA exposure; right panel: overlay of the pattern on the sagittal curvature map.
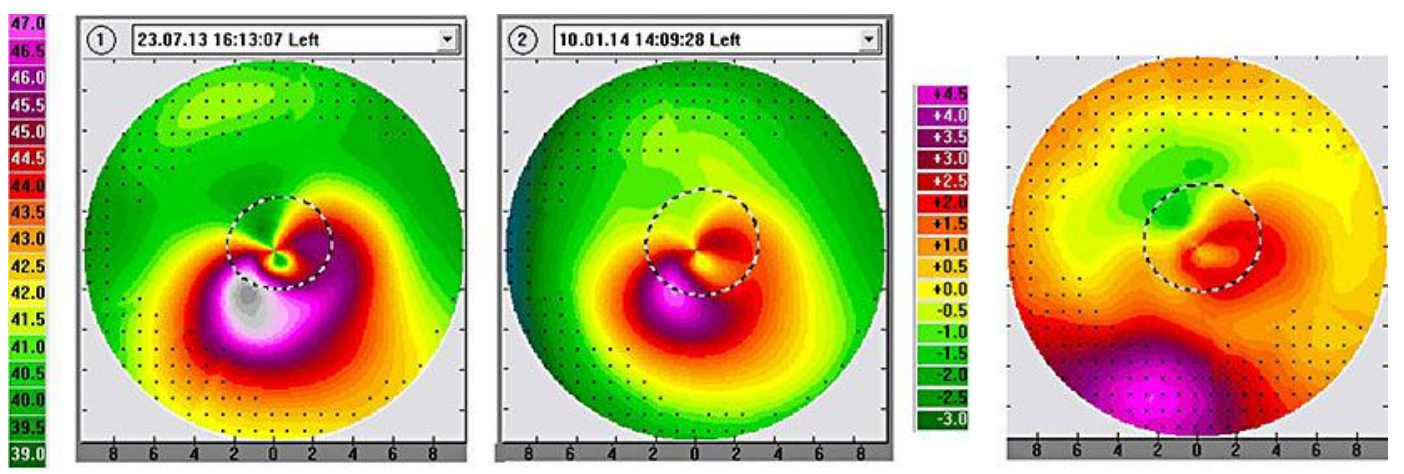

Fig. 2. Placido disk topography data showing sagittal curvature maps depicting significant refractive changes along the axis of the customized cross-linking pattern. Panel 1: 1 day preoperatively. Panel 2: 6 months postoperatively. Panel to the right: difference 2-1. 


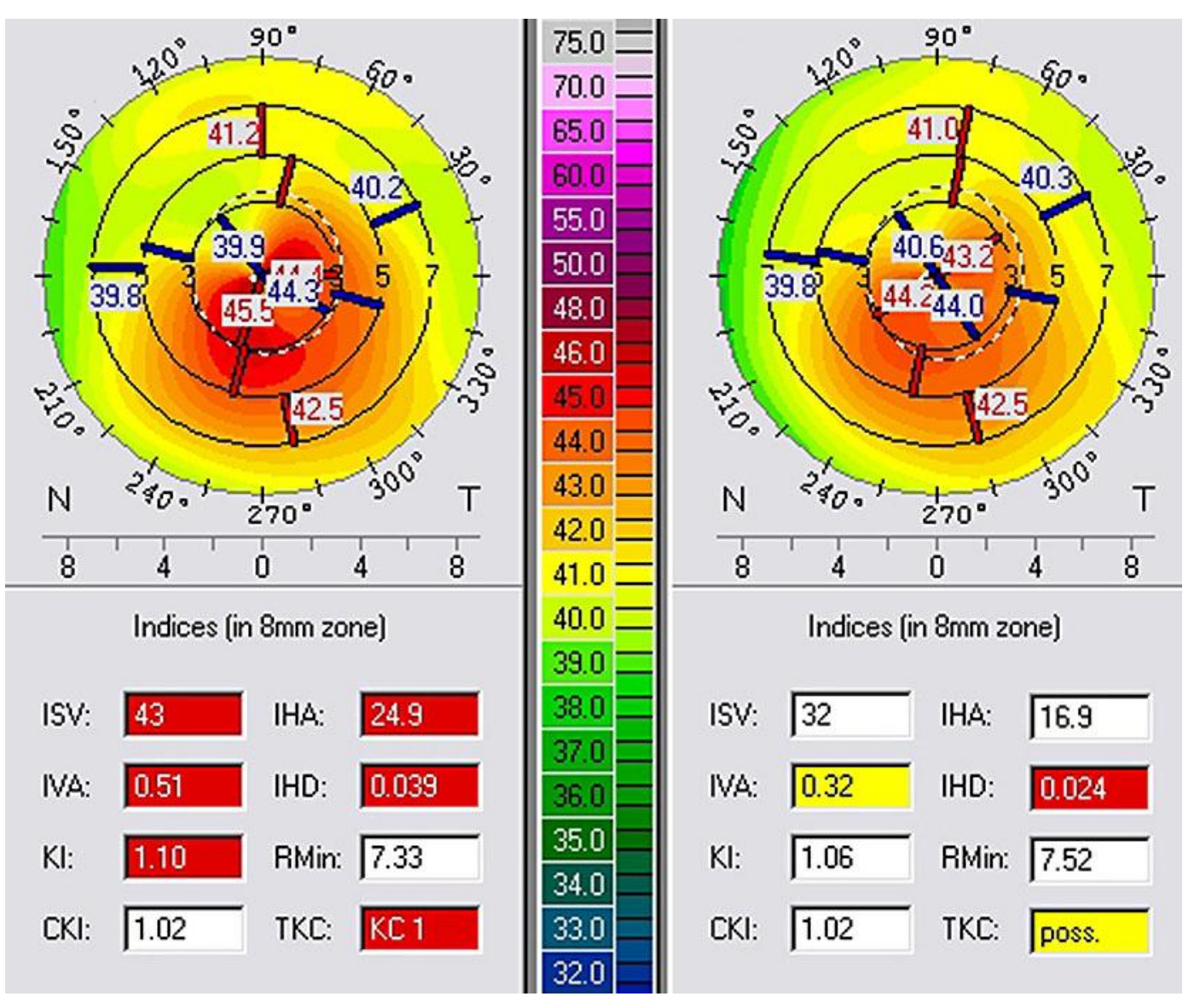

Fig. 3. Scheimpflug imaging data showing a comparison of preoperative versus postoperative data at 6 months depicting significant refractive changes along the axis of the customized cross-linking pattern as well as anterior surface normalization and ectasia arrest. 
Case Reports in

Ophthalmology

\begin{tabular}{l|l}
\hline Case Rep Ophthalmol 2014;5:172-180 \\
\hline DOI: 10.1159/000363371 & $\begin{array}{l}\text { @ 2014 S. Karger AG, Basel } \\
\text { www.karger.com/cop }\end{array}$ \\
\hline
\end{tabular}

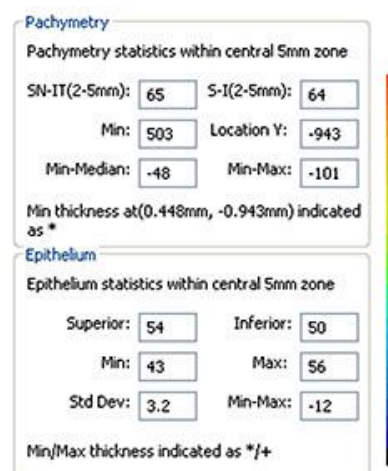

Mni/Max thickness indicated as */+

Kanellopoulos et al.: Topographically Customizable Toric Transepithelial CXL
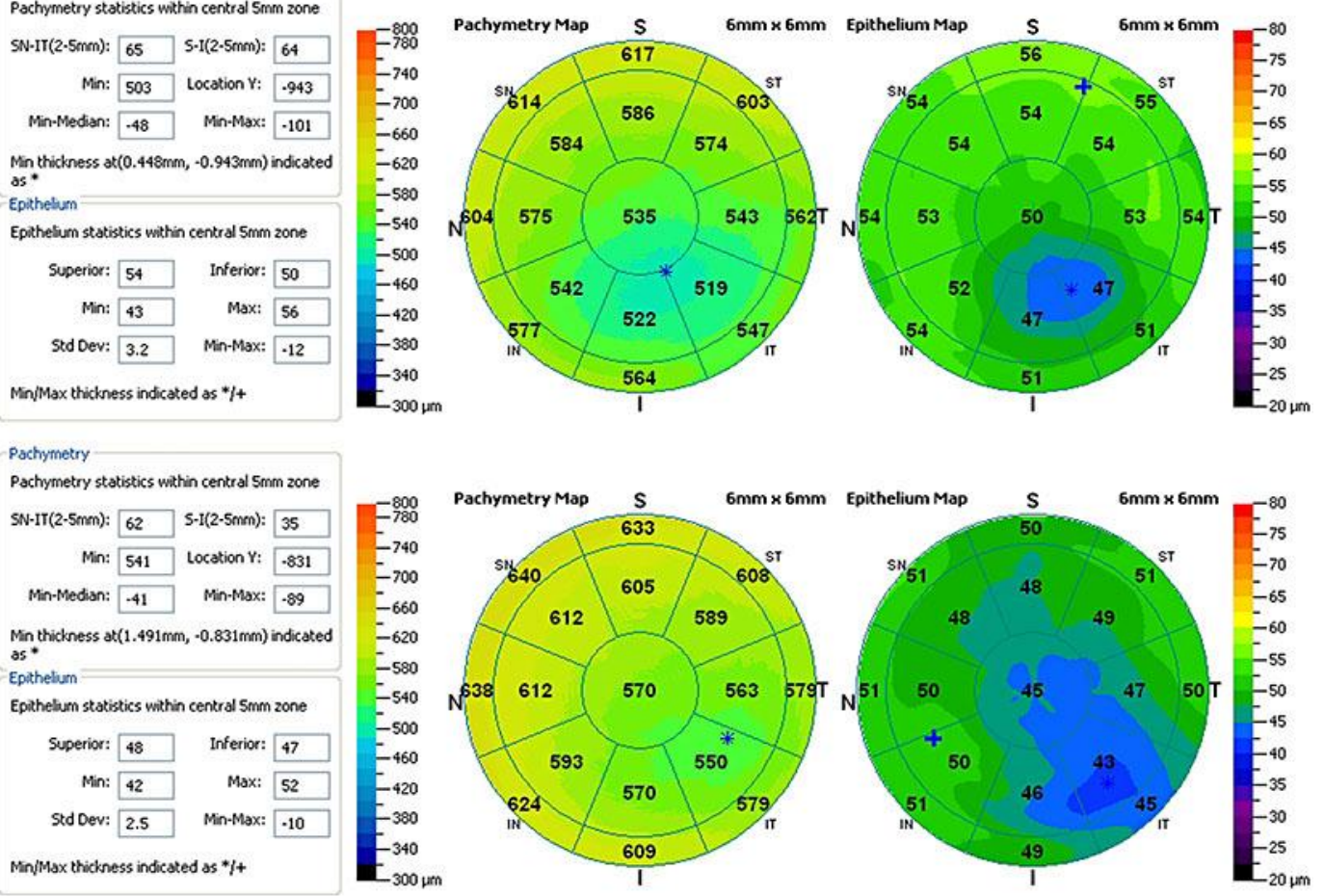

Pachymetry

Pachymetry statistics within central $5 \mathrm{~mm}$ zone

SN-IT(2-5mm): $62 \quad$ S-I (2-5mm): 54

Min: 528 Location Y: -902

Min-Median: $-44 \quad$ Min-Max: -94

Mn thickness at $(0.554 \mathrm{~mm},-0.902 \mathrm{~mm})$ indicated

as *

Epithelium

Superior: $53 \quad$ Inferior: 50

Min: 46

Max: 56

Std Dev: 2.5

Min-Max: -10

Mn/Max thickness indicated as */+
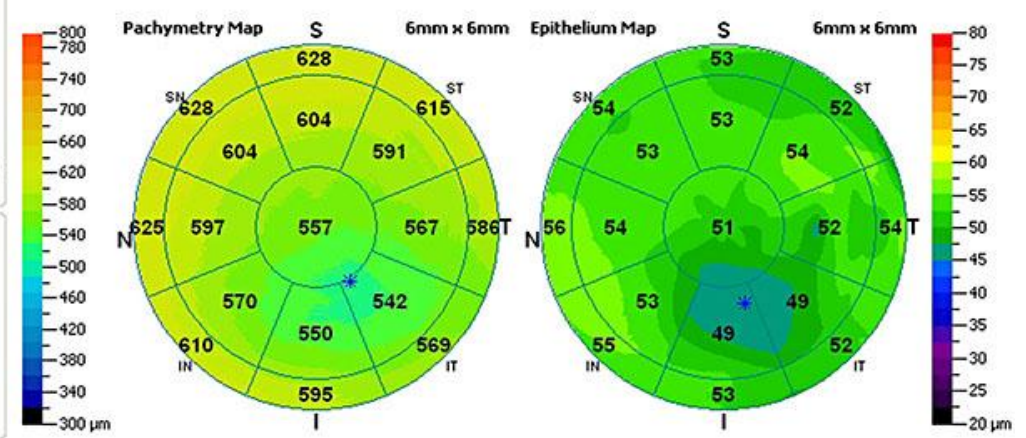

Fig. 4. Anterior segment OCT imaging pachymetry maps for the cornea (left) and corneal epithelium (right) covering the center of the 6-mm diameter area. Top panel: preoperatively; middle panel: 1-week postoperatively, and bottom panel: 6 months postoperatively. 